Supporting information for

\title{
Injectable and NIR-Responsive Hydrogels Encapsulating Dopamine- Stabilized Gold Nanorods with Long Photothermal Activity Controlled for Tumor Therapy
}

\author{
Jinfeng Zenga, Dongjian Shi*a, Yanglin Gu ${ }^{\mathrm{b}}$, Tatsuo Kaneko ${ }^{\mathrm{c}}$, Li Zhang ${ }^{\mathrm{a}}$, Hongji Zhang, ${ }^{\mathrm{a}}$ Daisaku \\ Kaneko a and Mingqing Chen*a
}

\footnotetext{
${ }^{a}$ Key Laboratory of Synthetic and Biological Colloids, Ministry of Education, School of Chemical and Material Engineering, Jiangnan University, Wuxi, China

b The Affiliated Wuxi No.2 People's Hospital of Nanjing Medical University

c Graduate School of Advanced Science and Technology, Japan Advanced Institute of Science and Technology, Ishikawa, 923-1292, Japan
}

corresponding author:

Dr. Dongjian Shi, djshi@jiangnan.edu.cn,

Prof. Mingqing Chen, mqchen@jiangnan.edu.cn 


\section{Characterizations of AuNRs-PDA}

AuNRs were prepared in two steps using seed-mediated growth as following: (1) $\mathrm{Au}$ nanoparticle (AuNP) seeds formation: Freshly prepared $\mathrm{NaBH}_{4}$ solution $(0.6 \mathrm{~mL}$, $0.01 \mathrm{M})$ was quickly added into a $\mathrm{HAuCl}_{4} \cdot 3 \mathrm{H}_{2} \mathrm{O}(0.25 \mathrm{~mL}, 0.01 \mathrm{M})$ and CTAB $(9.75$ $\mathrm{mL}, 0.1 \mathrm{M}$ ) mixed solution by vigorous stirring for $2 \mathrm{~min}$. After keeping the solution at room temperature for at least $2 \mathrm{~h}$, AuNPs (as seeds solution) were obtained. (2) Seeds growing: Growth of AuNRs was prepared by firstly mixing $\mathrm{HAuCl}_{4} \cdot 3 \mathrm{H}_{2} \mathrm{O}(0.5 \mathrm{~mL}$, $0.01 \mathrm{M})$ and $\mathrm{AgNO}_{3}(0.1 \mathrm{~mL}, 0.01 \mathrm{M})$ in $\mathrm{CTAB}$ solution $(10 \mathrm{~mL}, 0.1 \mathrm{M})$. After 0.08 $\mathrm{mL}$ ascorbic acid solution $(0.1 \mathrm{M})$ was added into the above solution, color of the mixed solution changed from yellow to colorless with blending completely. Followed by adding $0.2 \mathrm{~mL} \mathrm{HCl}$ solution $(1.0 \mathrm{M}), 0.024 \mathrm{~mL}$ seeds solution was also added into the resultant solution with gentle inversion for $10 \mathrm{~s}$. Finally, the solution was left stationary for AuNRs growing. AuNRs were purified by centrifugation for three times at 7500 $\mathrm{r} / \mathrm{min}$ for $10 \mathrm{~min}$ and kept for further use.

The purified AuNRs were then dispersed into $5 \mathrm{~mL}$ SH-PEG- $\mathrm{CH}_{3}$ aqueous solution $(2 \mathrm{mg} / \mathrm{mL}, \mathrm{Mw}=2000)$ with ultrasonic shaking for $3 \mathrm{~min}$ to completely disperse AuNRs, and then the dispersion liquid was stirred for $16 \mathrm{~h}$ at room temperature AuNRs-PEG was obtained by centrifuged three times at $7500 \mathrm{r} / \mathrm{min}$ for $10 \mathrm{~min}$. AuNRs-PEG was further coated with polydopamine (PDA) by dispersing AuNRs-PEG into $5 \mathrm{~mL}$ DA buffer solutions at room temperature for $30 \mathrm{~min}$.

X-ray photoelectric spectroscopy (XPS) of the AuNRs-PEG was employed to analyze the surface characteristic bonds of the nanorods via the binding energies, as shown in Figure S1. The analysis of the Au 4f spectrum showed a very broad signal, which was similar with the previously reported adsorption of sulfur on gold. Thereinto, two main peaks were observed for the $\mathrm{Au} 4 \mathrm{f}$ signal. The first peak located at $\sim 83.8 \mathrm{eV}$ was corresponding to $\mathrm{Au} 4 \mathrm{f}_{7 / 2}$, and a second doublet for $\mathrm{Au} 4 \mathrm{f}_{5 / 2}$ component was also observed at $\sim 87.6 \mathrm{eV}$, which indicated the existence of then $\mathrm{Au}-\mathrm{S}$ bond in the surface of AuNRs. 


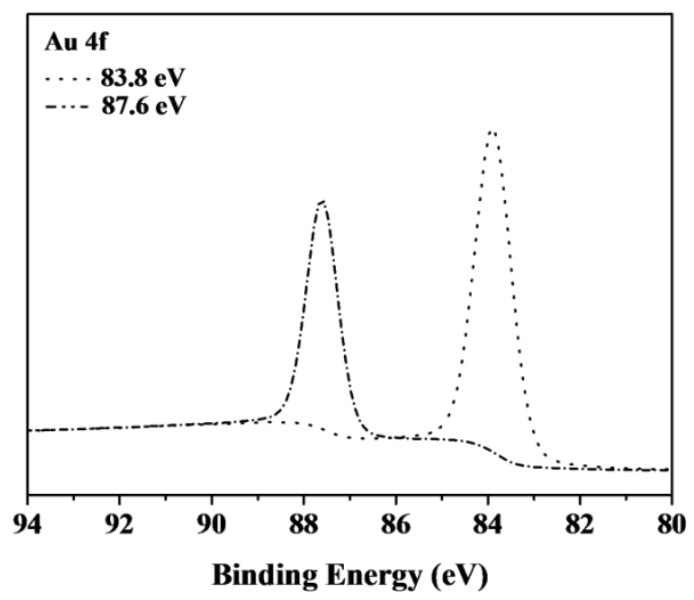

Figure S1. XPS analysis of AuNRs-PEG (Au $4 \mathrm{f}$ binding energies)

Raman spectrum of AuNRs-PEG by excited at $785 \mathrm{~nm}$ is shown in Figure S2. A Raman band at $310 \mathrm{~cm}^{-1}$ was contributed to the S-Au interaction, suggesting the coat layer formation of PEG-SH on the Au NRs.

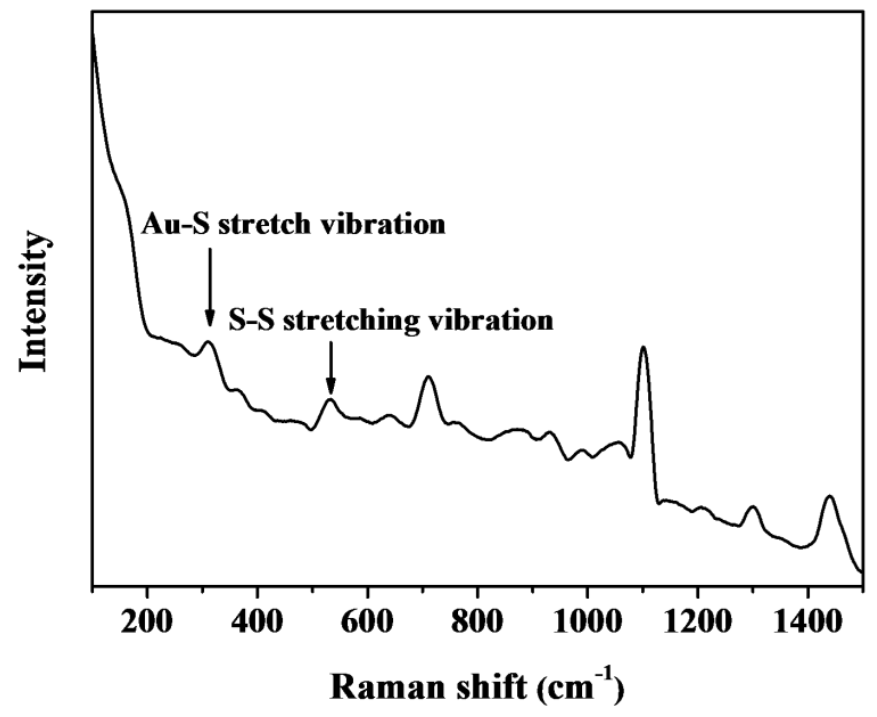

Figure S2. Raman spectrum of AuNRs-PEG with $785 \mathrm{~nm}$ of excitation

Diameters of the nanorods were determined by dynamic laser light scattering (Figure S3). The average hydrodynamic diameter of AuNRs-PEG in water was $82 \mathrm{~nm}$, which was similar with AuNRs $(70 \mathrm{~nm})$. After modification of PDA, the average hydrodynamic diameter of AuNRs-PDA ${ }_{0.1}$, AuNRs-PDA $_{0.25}$, AuNRs-PDA $_{0.5}$ were 101, 127, and $164 \mathrm{~nm}$, respectively. The average diameter and size distribution of Au NRsPDA were increased with various deposition thicknesses of PDA. 


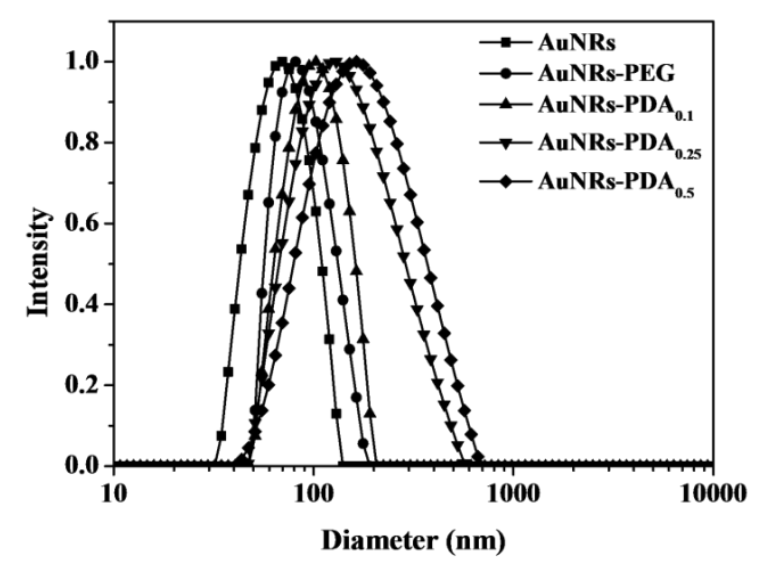

Figure S3. Hydrodynamic size distributions of Au NRs, Au NRs-PEG and Au NRs-PDA.

\section{Synthesis and characterization of dopamine-modified alginate (AIg-DA)}

Alg-DA was synthesized by grafting dopamine onto alginate by amidation reaction under the assistance of the EDC/NHS as described previously. Briefly, $2 \mathrm{~g} \mathrm{Alg}$ (10.1 mmol) was dissolved completely in $100 \mathrm{~mL}$ PBS buffer solution $(\mathrm{pH}=5.5,0.2$ M) under stirring vigorously. Then, EDC (20.1 mmol) and NHS (40.2 mmol) were added into the Alg aqueous solution to activate carboxylic groups on Alg molecules adequately for $45 \mathrm{~min}$. Subsequently, predefined amount of DA was added into the above mixture (Scheme S1). Double-rowed pipe was utilized for three times to prevent the oxidization of DA. And then, the above mixture was stirred for $24 \mathrm{~h}$ at room temperature under $\mathrm{N}_{2}$ protection. Finally, the mixture was precipitated and washed 3 times using ethanol. The final samples were dialyzed for 2 days against water, and followed by lyophilization. In order to obtain Alg-DA with various DA contents, different molar ratios of alginate to dopamine $(1: 1,1: 3,1: 5)$ were designed, noting as $\mathrm{Alg}-\mathrm{DA}_{1}, \mathrm{Alg}-\mathrm{DA}_{3}$, and Alg-DA, respectively.

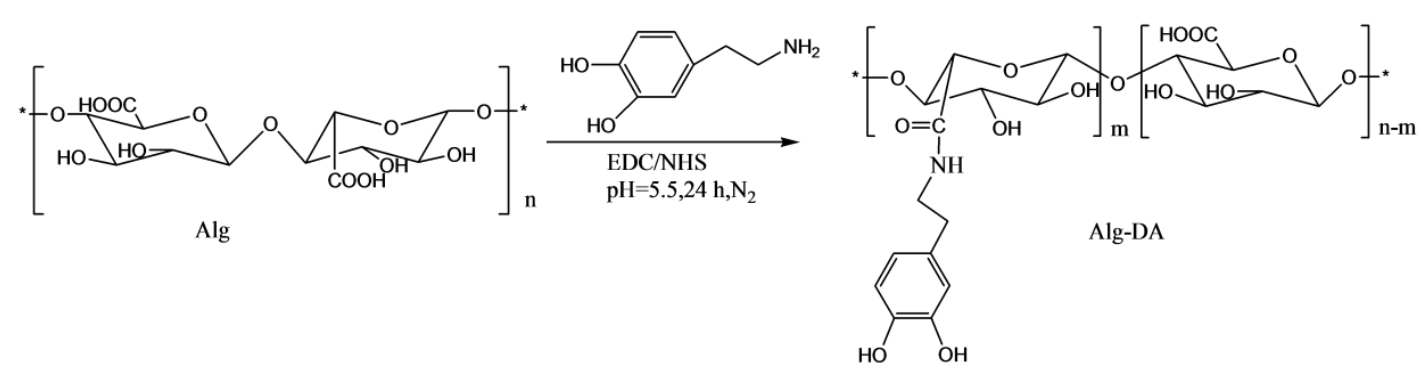

Scheme S1. Schematic representation of the synthesis process of dopamine modified alginate

(Alg-DA)

The structure analyses of Alg-DA were by FT-IR and ${ }^{1} \mathrm{H}-\mathrm{NMR}$ spectra (Figure 
S4a) and elemental analysis. Figure S4a shows FTIR spectra of Alg and Alg-DA with various DA contents. Characteristic absorption band at $1650-1800 \mathrm{~cm}^{-1}$ assigned to the $\mathrm{C}=\mathrm{O}(-\mathrm{COOH})$ in $\mathrm{Alg}$. After introducing DA, amide bonds $(\mathrm{C}=\mathrm{O})$ at $1730 \mathrm{~cm}^{-1}$ and $\mathrm{C}-\mathrm{O}$ stretch vibration band at $1253 \mathrm{~cm}^{-1}$ were found in Alg-DA, owing to the reaction between carboxylic acid groups of Alg and primary amino groups of DA and the phenol groups in DA. ${ }^{1} \mathrm{H}-\mathrm{NMR}$ spectra of Alg and Alg-DA with various DA contents were recorded in $\mathrm{D}_{2} \mathrm{O}(\delta 4.8)$ at room temperature and shown in Figure S4b. Chemical shifts at $\delta 3.0 \sim 6.0$ were assigned to the protons of the $\beta$-D-mannuronic and $\alpha$-L-guluronic units in Alg. The characteristic peak of benzene also appeared in the ${ }^{1} \mathrm{H}-\mathrm{NMR}$ spectra of Alg-DA at $\delta 7.33 \sim 6.93$. All the results from FTIR and ${ }^{1} \mathrm{H}-\mathrm{NMR}$ spectra suggested the successful introduction of DA into Alg.

(a)

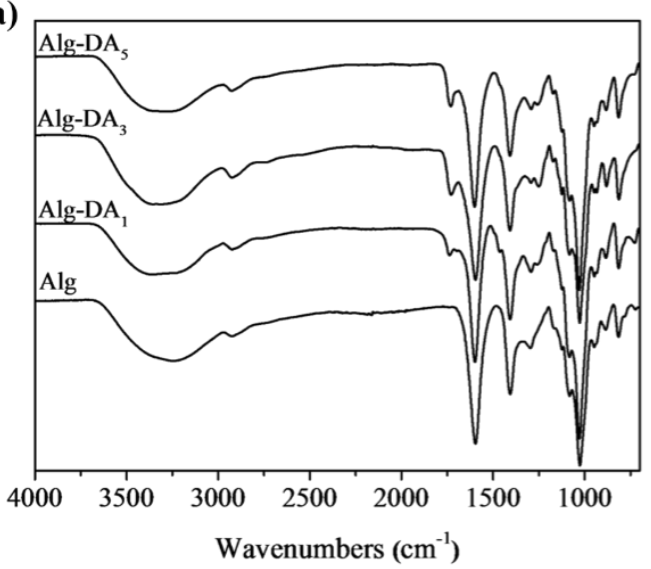

(b)

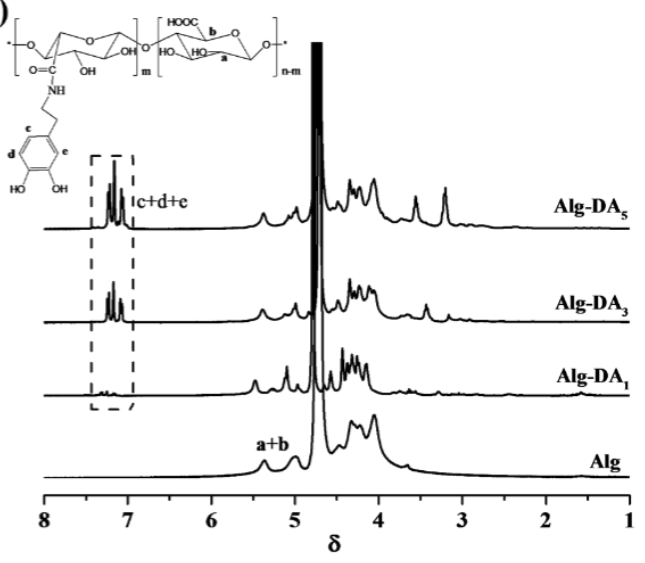

Figure S4. FTIR (a) and ${ }^{1} \mathrm{H}-\mathrm{NMR}$ (b) spectra of Alg and Alg-DA with various DA contents.

By comparison of the peak areas between the benzene groups $(\delta 7.33 \sim 6.93)$ and the methylene groups in Alg ( $\delta 4.8 \sim 5.7)$, the contents of DA in the Alg were 9.8\%, $33.0 \%$, and $47.3 \%$ (Table S1) for the feed molar ratios of DA to Alg at 1:1, 1:3 and 1:5, respectively. Moreover, element analysis was used to detect the DA contents, as listed in Table S1, which was similar with the results from ${ }^{1} \mathrm{H}-\mathrm{NMR}$ spectra. For clarity, these DA modified Alg polymers were abbreviated as Alg-DA $1, A l g-\mathrm{DA}_{3}$ and Alg-DA .

Table S1. Graft ratio of Alg-DA on ${ }^{1} \mathrm{H}-\mathrm{NMR}$ and elemental analysis.

\begin{tabular}{cccc}
\hline Molar ratio of Alg to DA & $1: 1$ & $1: 3$ & $1: 5$ \\
\hline${ }^{1}$ H-NMR (\%) & 9.8 & 33.0 & 47.3 \\
Elemental analysis(\%) & 9.0 & 27.9 & 49.8 \\
\hline
\end{tabular}




\section{Characterization of the hydrogel}

Figure S5 shows the gelation time of the CGP/Alg thermo-sensitive hydrogels with various mass ratios of CGP:Alg (5:0, 4:1, 3:1, 2:1, 1:1). The gelation time was decreased gradually from $40 \mathrm{~min}$ to $30 \mathrm{~min}$ and even to only 2 min with the increment of the Alg compositions, because of the hydrogen-bond and electrostatic interactions between CS and Alg.

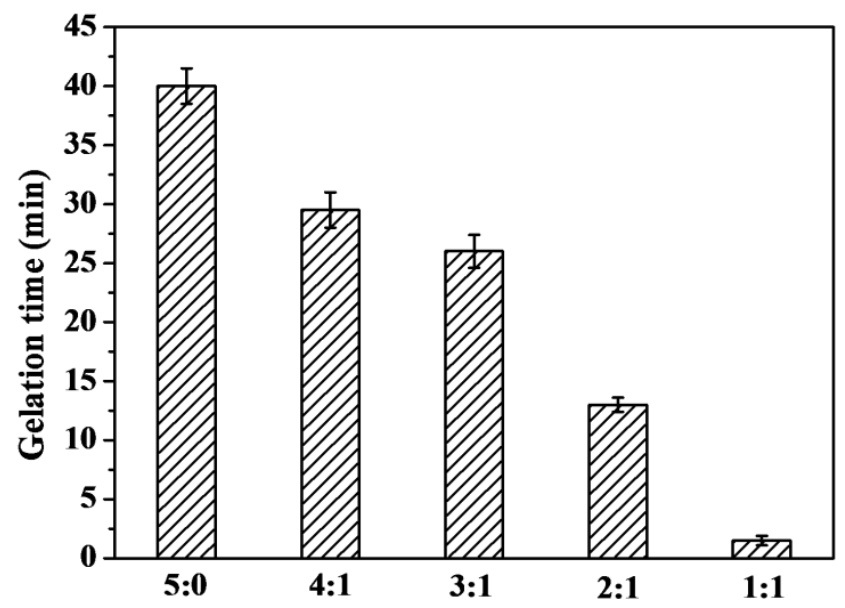

Figure S5. Gelation time of CGP/Alg thermo-sensitive hydrogel

Optical photographs showed that the CGP/Alg hydrogel with white color, and the CGP/Alg-DA/AuNRs nanocomosite hydrogels were dark red in the presence of PDA. Microtopography of the CGP/Alg-DA/AuNRs hydrogel was observed by SEM image (Figure S6). The CGP/Alg hydrogel and CGP/Alg-DA/AuNRs nanocomposite hydrogels showed almost the same pore structure, independing on the DA contents.
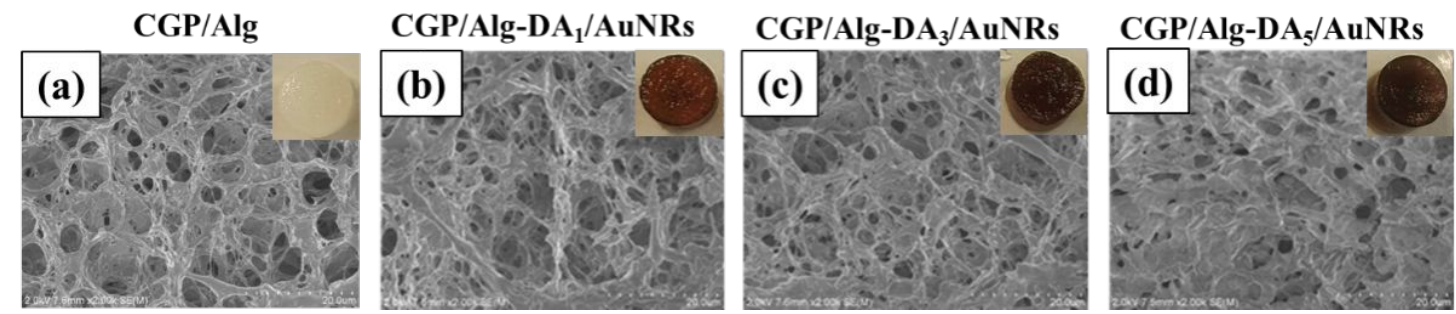

Figure S6. SEM images of CGP/Alg (a), CGP/Alg-DA 1 /AuNRs (b), CGP/Alg-DA 3 /AuNRs and

CGP/Alg-DA 5 /AuNRs composite hydrogels. The inset images are optical photographs of the hydrogels.

Figure S7 showed the UV-vis spectra of AuNRs and the supernatants of the CGP/Alg-DA/AuNRs gel after immersed in PBS buffer solution for 1 day and 2 weeks. AuNRs showed a special absorbance at about $800 \mathrm{~nm}$. However, there was no 
absorbance for the supernatant of the hydrogel even immersed after 2 weeks, indicating no AuNRs leaked from the CGP/Alg-DA/AuNRs composite hydrogel.
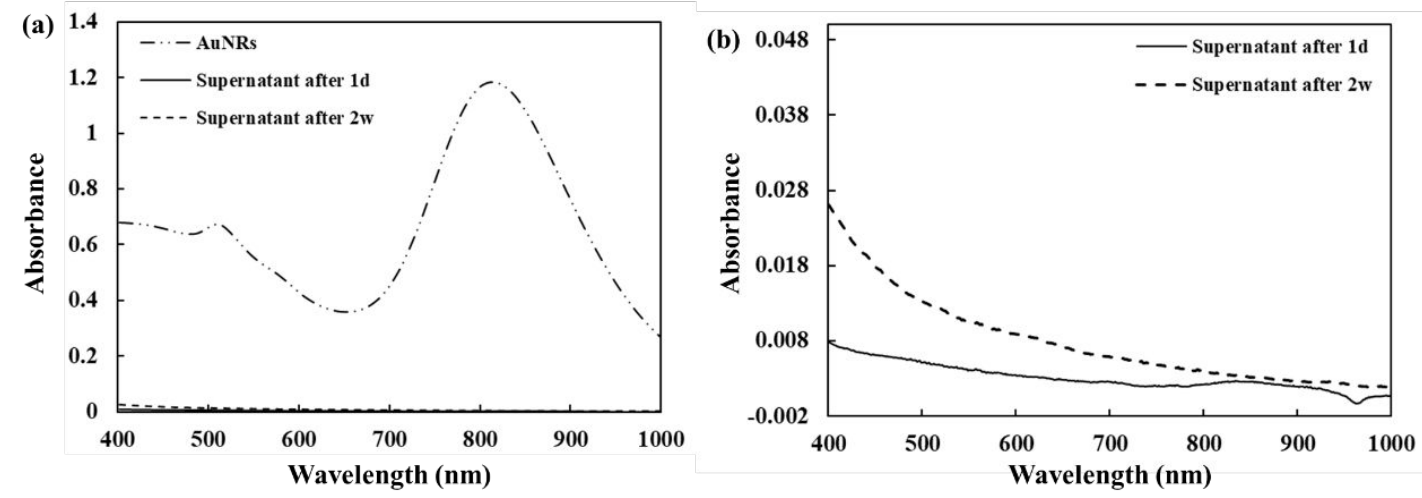

Figure S7. (a) UV-vis spectra of the AuNRs, the supernatant of the CGP/Alg-DA 1 /AuNRs composite hydrogels after immersed in PBS for 1 day and 2 weeks, and (b) the magnified UV-vis spectra of the upernatant of the CGP/Alg-DA $/$ AuNRs composite hydrogels after immersed in

PBS for 1 day and 2 weeks.

Compression strength of the hydrogels in the equilibrated swelling state was recorded at $60 \%$ strain with $1.0 \mathrm{~mm} / \mathrm{min}$ of compresses rate using a mechanical testing machine (WDW-1E, Jinan). The results were expressed as means of three parallel replicates, and shown in Figure S8a. The compression strength of the hydrogels was above $30 \mathrm{KPa}$ and increased to $63 \mathrm{KPa}$ with increasing DA contents. After immersing the hydrogel in PBS for 15 days, the strengths declined slightly, about $10 \%$ less than the original hydrogels. Since CS and Alg are biodegradable biopolymers, CGP/AlgDA/AuNRs hydrogels might also degrade in PBS, resulting in the decrement of compression strength. To confirm this presumption, the degradation behavior of the hydrogel was investigated in PBS (pH 7.4) at $37^{\circ} \mathrm{C}$. As shown in Figure S8b, within the first period of 2 weeks, the weights of the hydrogels declined around $10 \%$, and to almost half after 8 weeks. This weight loss was well corresponding to the declined strength of the hydrogels. Accordingly, the decreased strength was confirmed to be induced by the degradation of hydrogel, not the leakage of AuNRs. With prolonging the degradation time, the wight loss increased and to almost less than half of the wight after 8 weeks. For the pure CGP and CGP/Alg hydrogels, they almost completely degraded after 8 weeks. However, the weight of the CGP/Alg-DA/AuNRs hydrogels 
remained about $40 \% \sim 50 \%$ after 8 weeks, exhibiting lower degradation rate than the hydrogels without AUNRs-PDA. There might be two reasons: the one was undegradation of AuNRs, and another was the strong interactions between DA and polymers or PDA. Hence, the degradation of the hydrogels could be controlled to a suitable time to support AuNRs in the tumor site stably for high and long-time PTT efficacy.

(a)

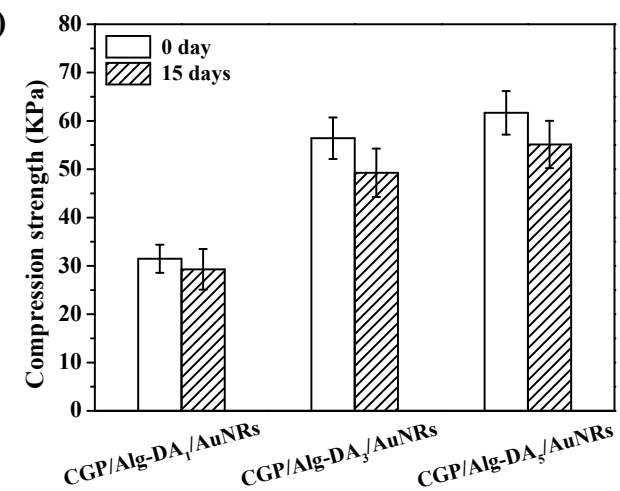

(b)

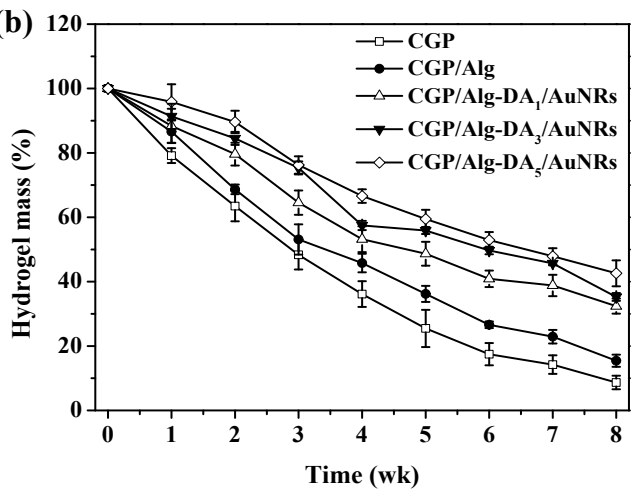

Figure S8. (a) Compression strengths of hydrogels treated before and after 15 days immersion. (b)

Degradation rate $\left(\mathrm{pH}=7.4,37^{\circ} \mathrm{C}\right)$ of CGP/Alg-DA/AuNRs hydrogel

The body weight was monitored as an indicator of physical health of mice during implantation of foreign material, laser irradiation and repeated PTTs, as shown in Figure S9. The mice in all groups maintained their normal growth characteristics without sharp reduction or increase in 21 days, suggesting that multiple PTTs were tolerated well with negligible side effects.

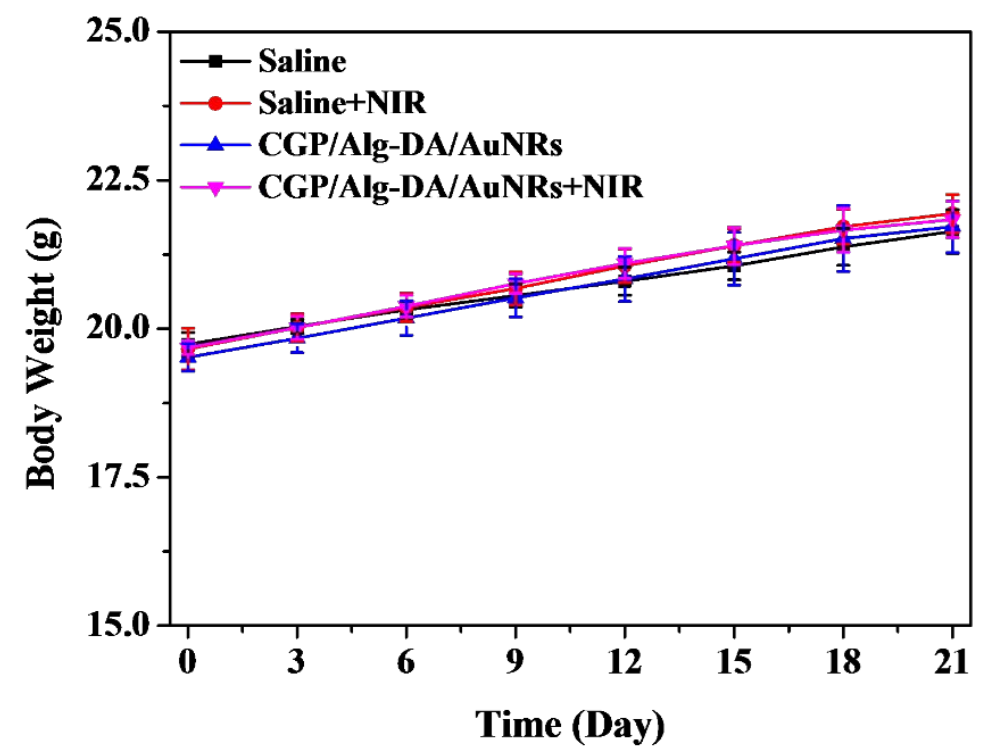

Figure S9. Body weight of HepG2-bearing Balb/c mice upon various treatments. 
